Universidad de Lima

Facultad de Psicología

Carrera de Psicología

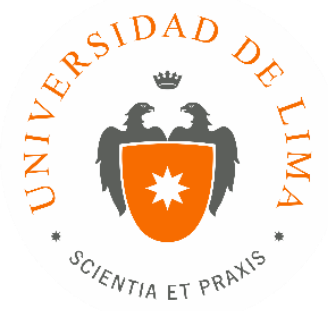

\title{
IMPLEMENTACIÓN DE PROCESOS E INDICADORES EN ÁREA DE CAPACITACIÓN
}

Trabajo de suficiencia profesional para optar el título profesional de Licenciado en Psicología

\section{Elio Andrés Bonifaz Terrones}

Código 20061391

Lima - Perú

Febrero de 2019 


\section{IMPLEMENTACIÓN DE PROCESOS E INDICADORES EN EL ÁREA DE CAPACITACIÓN}




\section{ÍNDICE GENERAL}

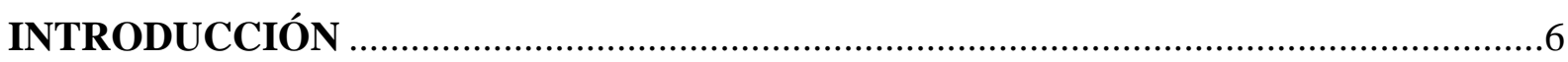

CAPÍTULO I: IDENTIFICACIÓN DEL PROBLEMA ………………………………….....

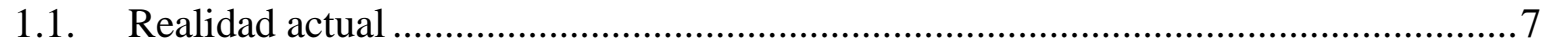

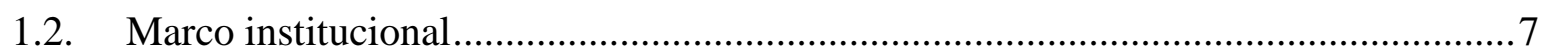

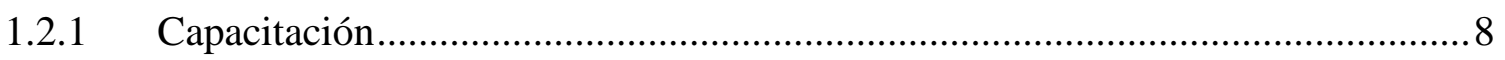

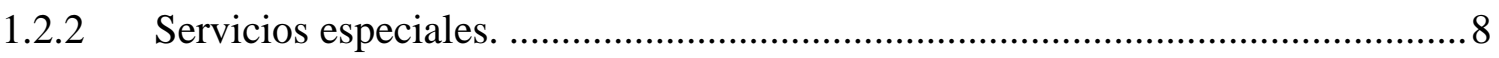

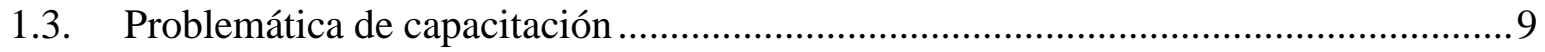

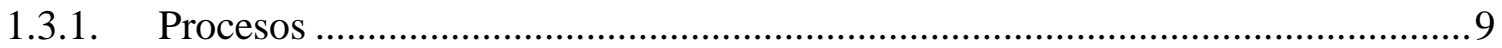

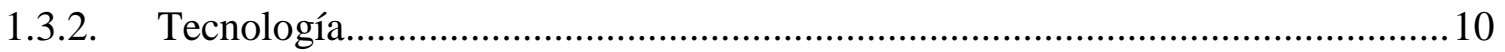

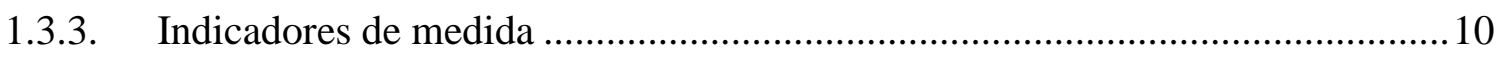

CAPÍTULO II: DESCRIPCIÓN DE LAS ACTIVIDADES Y TAREAS REALIZADAS12

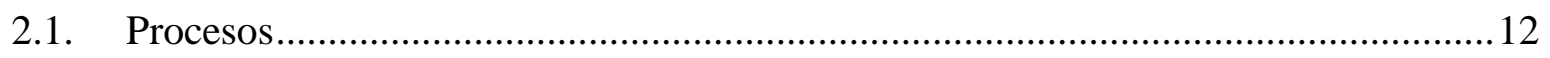

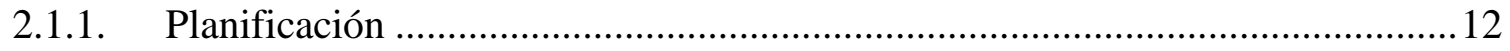

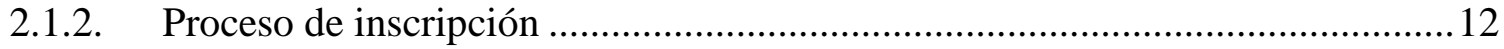

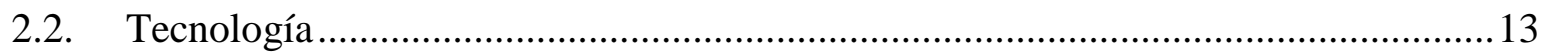

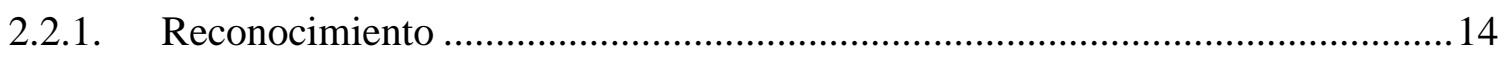

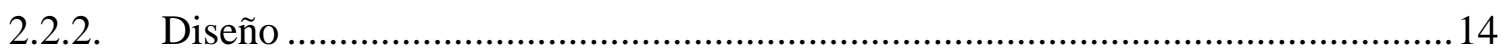

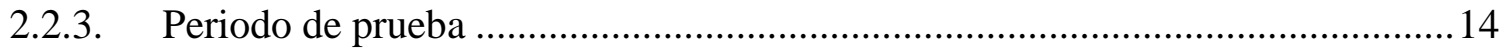

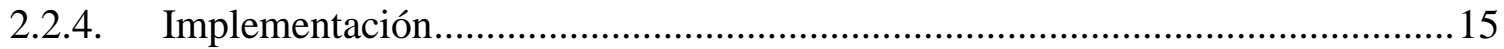

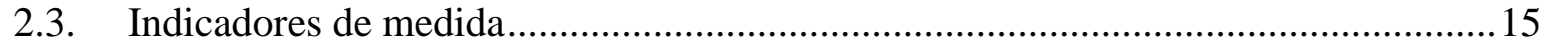

CAPÍTULO III: RESULTADOS DE LA INTERVENCIÓN.......................................16

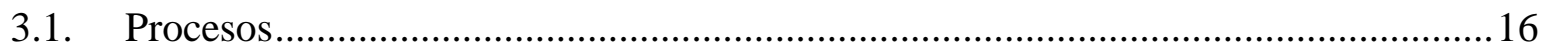

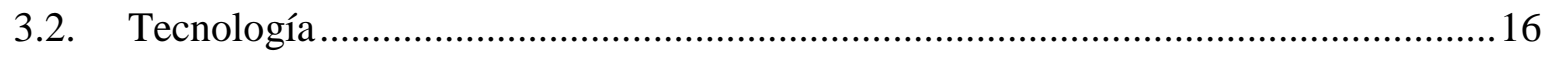

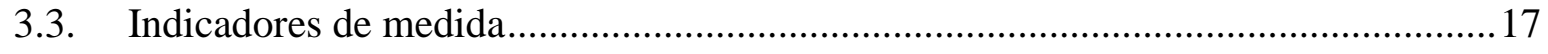

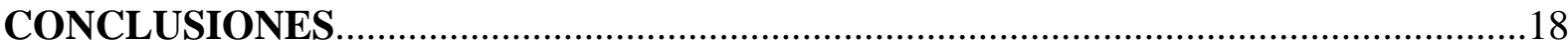

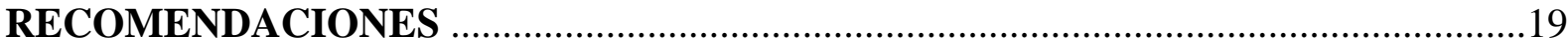

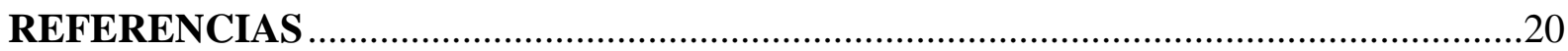




\section{ÍNDICE DE APÉNDICES}

Apéndice 1: Organigrama de recursos humanos .....................................................................22

Apéndice 2: Cadena de mando del departamento de servicios especiales .............................23

Apéndice 3: Diagrama de flujo de solicitud de cursos de capacitación ..................................24

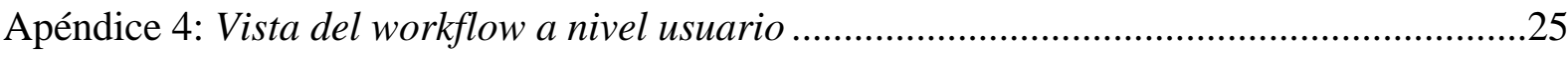

Apéndice 5: Indicadores de horas y dinero invertido en el 2017 ..........................................26 


\section{INTRODUCCIÓN}

Con el paso del tiempo la persona se ha convertido en el recurso más valioso de la organización. Ante ello, Recursos Humanos tiene el reto de promover su desarrollo y así poder capitalizar los conocimientos y habilidades para utilizarlo en favor de los resultados de la organización. Los cambios constantes en el contexto afectan directamente a la gestión de recursos humano, por estas razones se debe encontrar nuevas formas de ser más efectivo.

El objetivo del presente trabajo es desarrollar el caso de una empresa que muestra deficiencias en su gestión de la capacitación y las acciones de cambio que se propusieron para modificar el status quo. Con más detalle se busca explicar el contexto de la organización a fin de comprender su funcionamiento interno y desarrollar los tres problemas identificados: Procesos, Tecnología e Indicadores.

Los procesos hacen referencia a la falta de orden y organización de las responsabilidades que tiene a cargo capacitación, por tecnología se entiende la falta de un software de apoyo que facilite el orden y recolección de la información, por último, la falta de indicadores que nos permitan medir y evaluar la situación actual y de poder desarrollarlos se podría obtener una ayuda visual para alcanzar a tomar decisiones más rápido y de manera oportuna.

Para cada una de estas dificultades se desarrolla un plan de acción y finalmente se presentan los resultados cuantitativos y cualitativos obtenidos a lo largo del proceso. 


\section{CAPÍTULO I: IDENTIFICACIÓN DEL PROBLEMA}

\subsection{Realidad actual}

Las organizaciones han ido reconociendo que las personas son un factor determinante para el logro sus objetivos e importantes en su supervivencia ante futuras adversidades. Chiavenato (2017) menciona que el capital humano está conformado por las personas, sus talentos y habilidades, que debe ser aprovechado por las organizaciones para buscar nuevas soluciones y productos, recordando siempre que el capital intelectual aumenta constantemente y no se deprecia. Apoyando esta opinión, según la revista "Gestión de RRHH en América Latina" publicada por Deloitte en abril del 2018, una gestión estratégica reconoce que el capital más valioso que tiene una empresa son los conocimientos, habilidades y creatividad de sus trabajadores, y son ellos los responsables de los resultados de la organización.

Asimismo, Chiavenato (2017) explica que Recursos Humanos, ante este contexto en constante cambio, debe tener la capacidad de poder brindar a la organización las personas con conocimiento, habilidades y competencias que esta necesita, y podrá ofrecerlas en la medida que se gestione adecuadamente el capital humano. Es evidente que encontramos fuerzas cambiantes en el entorno, en las organizaciones y en los mismos individuos, son estas fuerzas las que modelan la forma en cómo se gestionan los recursos humanos.

La única forma de evaluar dicha gestión de recursos humanos es midiéndola, utilizando indicadores que nos permitan identificar el estado de los procesos internos, que permitan hacer seguimiento y evaluar su desempeño.

\subsection{Marco institucional}

El presente trabajo se desarrolla en una empresa transnacional dedicada a la comercialización de repuestos industriales y a brindar servicios especializados a diferentes clientes en todo el país. La empresa cuenta con 410 trabajadores, de los 
cuales se encuentran laborando en los diferentes departamentos de finanzas, operaciones, comercial, servicios especiales y recursos humanos. Se describen con más detalle a continuación el área de capacitación y el departamento de servicios especiales.

\subsubsection{Capacitación}

El departamento de recursos humanos esta compuesto por las áreas de reclutamiento y selección, bienestar social, nóminas, comunicación interna y capacitación (vea Apéndice 1 para mayor información). El área de capacitación tiene la responsabilidad de organizar todos los cursos dirigidos al personal técnico del departamento de servicios especiales, por esta razón, a principios de año propone un cronograma de cursos para todo el año. Por supuesto, cada líder puede inscribir a su personal a los cursos que él considere conveniente.

\subsubsection{Servicios especiales.}

Dentro del departamento de servicios especiales se tiene registro de aproximadamente 310 trabajadores que representan un total de $70 \%$ de la fuerza laboral, los cuales en su mayoría son personal técnico que laboran en unidades en Lima y provincias. Dentro de este grupo tenemos a los técnicos, líderes y supervisores y un gerente.

Se considera una unidad a una empresa industrial al cual se le ofrece servicios especiales de forma recurrente todos los meses, algunos de estos servicios son diagnóstico, corrección, mantenimiento de maquinaria industrial, entre otros. Actualmente se encuentran activas 18 unidades tanto en Lima como en diferentes provincias dentro de todo el Perú. Se tienen cuatro supervisores de los cuales cada uno tiene asignado entre dos a tres unidades. Además, cada unidad es comandada 
por un líder, los cuales tienen a cargo alrededor de 20 a 50 técnicos. Se describe gráficamente la cadena de mando en el Apéndice 2.

\subsection{Problemática de capacitación}

El departamento de servicios especiales tiene alrededor del $76 \%$ de la fuerza laboral, que, debido a un crecimiento desmesurado, actualmente cuenta con una pobre gestión de la capacitación. Se establecen tres dificultades que impiden una apropiada administración y se designan con las siguientes etiquetas: Procesos, Tecnología e Indicadores de medida. A continuación, se explica con más detalle cada uno de ellos.

\subsubsection{Procesos}

Por un lado, no se tiene esclarecido el procedimiento para realizar las inscripciones a los cursos, este debería estar definido de forma clara y debe ser difundido a las personas de interés. Por otro, la responsabilidad de inscribir al personal técnico recae sobre el líder de cada unidad de servicios especiales, ellos realizan las inscripciones progresivamente desde diez días antes del evento hasta incluso un día antes de la capacitación. Esto ha generado dificultades al asistente de capacitación que tiene que lidiar con inscripciones de último minuto que obligan a dedicar más tiempo del requerido a la organización de los cursos, además se han tenido que cancelar algunos cursos con muy poco tiempo de anticipación en los que no se contaba con un mínimo de participantes. Garnica (2013) resalta que dentro de las organizaciones existe una gran diversidad de información, actividades y actores que influyen dentro de ella, por ello se debe dedicar esfuerzos en establecer procesos de coordinación, de lo contrario generarán un ambiente de incertidumbre y falta de control, por otro lado, sugiere que las herramientas para combatirlo son la comunicación, una autoridad, supervisión y la proposición de normas y estándares. 


\subsubsection{Tecnología}

En este momento no se cuenta con ningún software de apoyo para las inscripciones mencionadas, para poder realizarlas se requiere de una comunicación formal vía correo electrónico que empieza desde solicitar información sobre los cursos, luego pedir las aprobaciones al jefe inmediato y finalmente notificar al asistente de capacitación. Los registros indican que se utilizan aproximadamente 150 correos por mes para este propósito, y debido al uso de este medio de comunicación, la trazabilidad de la información se dificulta. Cuesta (2010) menciona que recursos humanos va necesitando de nuevas tecnologías que la ayuden a procesar la información que tiene de manera automática para así permitir una mejor tomar de decisiones, una mejor organización laboral y promover acciones de mejora. Apoyando la misma idea, Dessler (2014) manifiesta que los sistemas de información permiten a Recursos Humanos agilizar sus procesos y mejorar el desempeño en su gestión y permite a los gerentes centrarse en aspectos más estratégicos.

\subsubsection{Indicadores de medida}

La información de los asistentes a los cursos se encuentra dispersa en diferentes hojas de cálculo de Excel y hojas de papel, dificultando así el análisis de la información. Además, la gerencia de servicios ha solicitado en oportunidades reportes que el asistente de capacitación puede demorar hasta 5 días hábiles en poder responder. Actualmente no se cuentan con indicadores que nos permitan evaluar cual es la situación actual. Mazabel (2015) resalta que los indicadores permiten observar rápidamente los resultados y poder compararlos con el planeamiento actual, permitiendo, además, poder tomar acciones correctivas en 
caso de ser necesario. Es decir, que tener acceso a indicadores permitiría evaluar el contexto actual y poder tomar decisiones en base a eso. 


\section{CAPÍTULO II: DESCRIPCIÓN DE LAS ACTIVIDADES Y}

\section{TAREAS REALIZADAS}

A continuación, se presentan las acciones que se realizan con la finalidad de resolver cada uno de los problemas que impiden tener una adecuada gestión de la capacitación.

\subsection{Procesos}

El objetivo es organizar los procesos de los cuales el área de capacitación es responsable.

\subsubsection{Planificación}

Las inscripciones de último minuto y las cancelaciones de cursos obligan al asistente de capacitación a invertir más tiempo en estas actividades, por ello, se busca que las inscripciones a los cursos se realicen de forma planificada. De ser así, el asistente de capacitación puede gozar de más tiempo para otras de sus actividades y los técnicos que buscan acceder al conocimiento puedan hacerlo en el momento que lo requieran. Ya identificado el problema, se propone que cada líder pueda enviar la lista de las personas con los cursos que llevarán a lo largo del año. Dicha lista deberá ser enviada hasta máximo finales de enero. Antes de implementar la lista, se busca sensibilizar a los líderes de los beneficios que traerá la planificación. Finalmente se comunica de forma oficial a todo el departamento de servicios especiales del cambio.

\subsubsection{Proceso de inscripción}

Para poder desarrollar el flujo del proceso de inscripciones primero se investiga sobre los componentes y actividades que lo conforman, para ello es necesario realizar una reunión con de la especialista de capacitación y la participación de dos 
líderes locales. Esta única reunión tiene como fin alinear las ideas que ambas áreas tienen del proceso e involucrarlas en el del diseño del mismo.

Gracias a la información recabada es posible diseñar dicho flujo utilizando el programa "Visio". Se presenta una versión a las áreas interesadas con el fin de lograr las correcciones necesarias para luego realizar las modificaciones y así obtener el producto final que se puede apreciar en el Apéndice 3. Finalmente, el proceso se comunica a los líderes, supervisores y gerencia de servicios especiales por medio de correo electrónico a fin de que todos sigan el procedimiento y se cumpla tal cual esta normado de ahora en adelante.

\subsection{Tecnología}

El objetivo es desarrollar un "workflow" o flujo de trabajo digital que permita automatizar las inscripciones a los cursos para poder disminuir la carga laboral del asistente de capacitación y nos permita recopilar toda la información del proceso.

Para ello se utiliza un software del Office 365 llamado "Sharepoint", este software genera un intranet al cual todos los colaboradores de la empresa pueden ingresar, una ventaja de usar este software es poder diseñar aquí un "workflow" o flujo de trabajo. Un "workflow" es una serie de procesos automatizados, que en el siguiente proyecto tiene una secuencia de tres pasos. En el primer paso, el líder de cada unidad tiene que inscribir a la persona que asistirá ingresando la información se le solicita, luego el "workflow" solicita la aprobación del jefe inmediato y finalmente, cuando el jefe inmediato responde, en ese momento el "workflow" notifica a la persona que se inscribió, al jefe inmediato y al área de capacitación que la inscripción se realizó con éxito. Toda la información recabada en este proceso es enviada a una base de datos con acceso limitado estrictamente al área de capacitación para mantener toda información personal bajo reserva. 
El proyecto se desarrolla en cuatro etapas: Reconocimiento, Diseño, Periodo de prueba, Implementación.

\subsubsection{Reconocimiento}

Se identifican los "stakeholders" o áreas interesadas en el proyecto, las cuales son el área de capacitación, el departamento de servicios especiales y la encargada de transformación digital. A continuación, se programan entrevistas con cada uno de ellos para identificar sus intereses frente al proyecto y para buscar involucrarlos con el mismo. Durante las entrevistas se pudo identificar la necesidad de tener indicadores que puedan medir la inversión en dinero y las horas capacitadas a cada unidad. De igual forma se determina como siguientes pasos el diseñar el flujo de inscripciones de capacitación para luego poder esbozar la herramienta digital con ayuda de la responsable de transformación digital.

\subsubsection{Diseño}

Anteriormente ya se ha explicado cómo se diseña el flujo de inscripciones, esta información es presentada a la encargada de transformación digital para poder trabajar en conjunto y elaborar un bosquejo del "workflow". Con toda la información reunida, la encargada de transformación digital se compromete a entregar el "workflow" en dos semanas.

\subsubsection{Periodo de prueba}

Ya listo el "workflow" se decide implementarlo en una unidad de servicios especiales, la unidad elegida tiene 20 trabajadores y el líder tiene una buena relación con el área de capacitación. El fin es revelar la calidad del nuevo proceso digital e identificar posibles errores. Se conversa con el líder a cargo para comentarle que se le brindará todo el soporte que necesite y explicarle como funciona este nuevo proceso de inscripciones a los cursos. Gracias a ello, se 
encontraron algunos errores de programación que fueron resueltos sin mayores inconvenientes y finalmente se presentan los avances a los "stakeholders" para que puedan ver los avances.

\subsubsection{Implementación}

Antes de implementar el proyecto, se decide guiar a los líderes en el uso del “workflow” para explicarles cómo funciona y las ventajas que obtendrán de este. Se realizan llamadas telefónicas con cada uno de los líderes para explicar el procedimiento y también se les hace llegar un folleto con imágenes con la misma información. Se implementa el proyecto en todas las unidades y se comunica mediante correo electrónico que esta será la única forma de inscribir a las personas en cursos de capacitación. Se muestran algunas imágenes del "workflow en el Apéndice 4.

\subsection{Indicadores de medida}

El objetivo es implementar indicadores que nos permiten evaluar el impacto que tiene la capacitación en el departamento de servicios especiales.

Gracias al proyecto que permitió digitalizar las inscripciones, se tiene acceso a una base de datos con información como: nombre del técnico, unidad a la que pertenece, nombre del curso, horas del curso y costo del curso. Con esta información se puede obtener dos indicadores: cantidad de horas de capacitación por unidad por mes y dinero invertido en dólares por unidad por mes, asimismo, se asigna como responsable de ellos al asistente de capacitación. Esta misma información puede ser graficada para ayudar a resumir visualmente la situación actual. Se muestra en el Apéndice 5 información de todo el departamento de servicios especiales ya que por motivos de confidencialidad no se puede mostrar los detalles por unidad. 


\section{CAPÍTULO III: RESULTADOS DE LA INTERVENCIÓN}

A continuación, se describen los resultados cualitativos y cuantitativos de las acciones desarrolladas en el capítulo anterior.

\subsection{Procesos}

Se logró conocer la cantidad de personas que asistirán a los cursos durante el año permitiendo identificar los cursos con alta demanda y también los que registran pocos asistentes.

A partir de aquí fue posible planificar y tomar mejores decisiones como la apertura de nuevas fechas para aquellos cursos con mayor demanda, acoplar cursos con pocos participantes para que no sea cancelado, y cerrar de manera definitiva los cursos con muy poco interés del público. Se reciben comentarios por parte de los líderes que el área de capacitación se organiza mejor y que ahora buscar involucrarse mucho más con ellos.

Asimismo, poder mostrar gráficamente en pasos secuenciales un procedimiento que se repite continuamente, permite tener una lectura fácil para quien necesite inscribir a una persona en una capacitación. Las llamadas y correos de los líderes preguntando sobre el procedimiento para inscribir una persona en un curso disminuyeron dramáticamente una vez comunicado el flujo.

\subsection{Tecnología}

Generó un ahorro de tiempo para todas las partes involucradas, además de brinda la posibilidad de que la información tenga una trazabilidad ofreciendo así la capacidad de controlar y dar seguimiento a las inscripciones. Tener acceso a información en tiempo real genera la sensación de que el área de capacitación está informada de lo que sucede con detalles. 
Se evidencia en los registros de correos electrónico que estos disminuyen en un $80 \%$ después de un mes implementado el "workflow".

\subsection{Indicadores de medida}

Tenemos al alcance una base de datos con información de las personas que han llevado alguna capacitación, dicha información nos permite generar los indicadores de horas y dinero invertido por cada unidad de servicios especiales.

Esta información se convierte en gráficos de fácil lectura permitiendo tomar mejores decisiones e identificando anormalidades. Uno de los casos encontrados es el de una unidad que destinó más de USD\$10,000.00 en un periodo de seis meses para capacitar al personal aduciendo que era necesario para mejorar su desempeño, sin embargo, este no aumentó, lo que inició una investigación más a profundidad.

Además, el tiempo de respuesta de los reportes solicita gerencia han pasado de tener una demora de cinco días a tan sólo un día, cinco veces más rápido. 


\section{CONCLUSIONES}

- Los procesos del departamento de Recursos Humanos tienen que ser definidos con claridad para poder tener control sobre los mismos. Para lograrlo es importante proponer normas y lineamientos, los cuales deben ser comunicados tanto de manera interna como externa del área.

- Las nuevas tecnologías tienen un rol importante en la gestión de recursos humano porque permiten organizar la gran cantidad de información que se genera continuamente. De esta forma se pueden automatizar los procesos operativos y concentrar sus recursos en temas que puedan agregar valor a la organización.

- Implementar indicadores en recursos humanos significa poder medir de forma objetiva la realidad actual, nos permite evaluar lo que sucede en el ahora para tomar mejores decisiones en el futuro. Especialmente si nos permite evitar gastos innecesarios y utilizar los recursos de manera más eficiente. 


\section{RECOMENDACIONES}

A continuación, se detallarán las recomendaciones:

- El departamento de Recursos humanos debe establecer con detalles sus procesos más importantes para poder tener control sobre ellos e identificar posibles acciones de mejora. Para lograrlo se debe proponer las normas y reglas que sean convenientes y comunicarlo adecuadamente tanto dentro como fuera del área.

- El departamento de Recursos humanos debe invertir tiempo y recursos para poder implementar las nuevas tecnologías y así reducir significativamente la carga operativa para que pueda dedicarse a realizar actividades que brinden valor a la organización.

- El área de capacitación debe continuar en la búsqueda de nuevos indicadores que le permitan continuar mejorando su gestión y calidad del servicio, por ejemplo, medir la satisfacción de los cursos que brinda. 


\section{REFERENCIAS}

Chiavenato, I. (2017). Administración de recursos humanos: el capital humano de las organizaciones.. México: Comercializadora de impresos OM.

Cuesta, A. (2010). Gestión del talento humano y del conocimiento. Bogotá: Ecoe Ediciones.

Deloitte (Abril, 2018). Gestión de RRHH en América Latina: compensaciones salariales, perfiles y retos del área en la Región. [versión PDF]. Recuperado de: https://www2.deloitte.com/content/dam/Deloitte/ec/Documents/humancapital/Gestion\%20de\%20RRHH\%20en\%20America\%20Latina.pdf

Dessler, G. (2017). Administración de recursos humanos: enfoque latinoamericano. México: Pearson Educación.

Garnica, A. (Octubre, 2013). La Importancia de los Procesos y Mecanismos de Coordinación en la Empresa: una aproximación a partir de la teoría de la organización. [versión PDF]. Recuperado de: https://www.researchgate.net/publication/287206214_La_Importancia_de_los_Proce

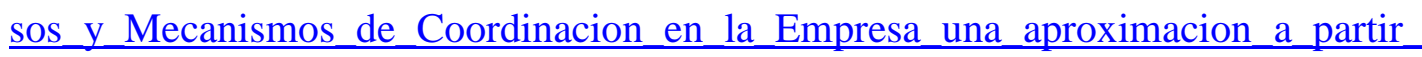
de_la_teoria_de_la_organizacion

Mazabel, G. C. (2015), Indicadores de recursos humanos y su resultado económico en la empresa: “de lo cualitativo a lo cuantitativo”. Lima: Centro de investigación de recursos humanos. 


\section{APÉNDICES}




\section{APÉNDICE 1: ORGANIGRAMA DE RECURSOS HUMANOS}

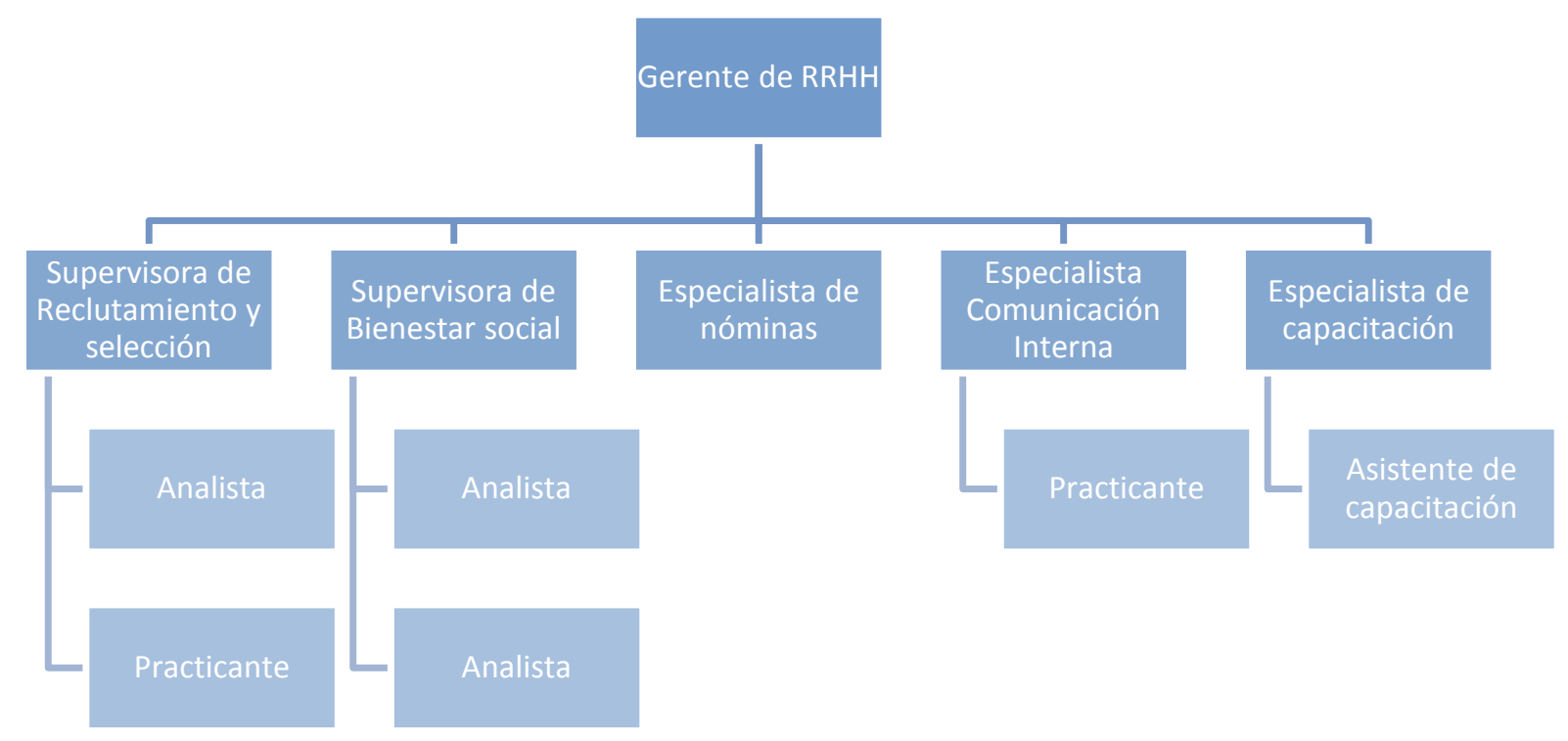




\section{APÉNDICE 2: CADENA DE MANDO DEL DEPARTAMENTO DE SERVICIOS ESPECIALES}

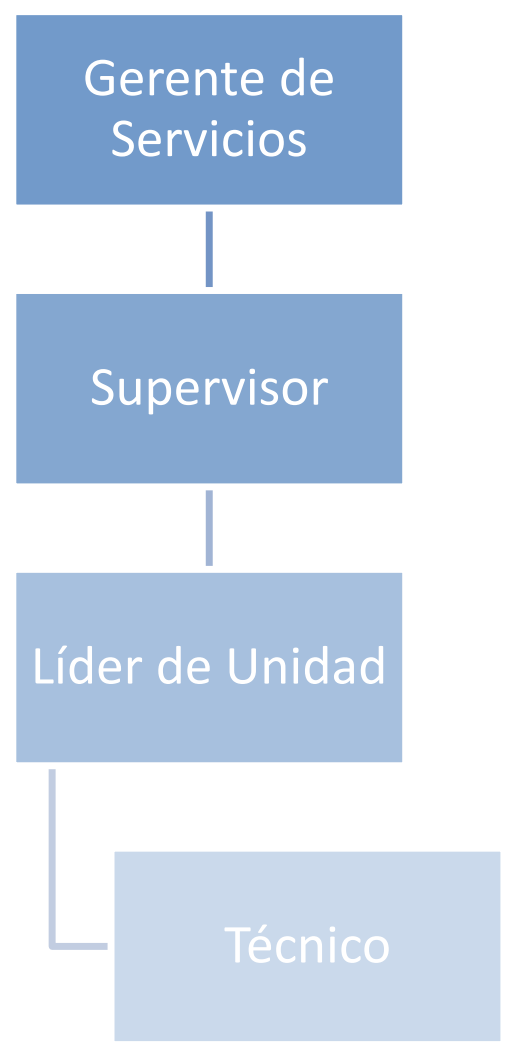




\section{APÉNDICE 3: DIAGRAMA DE FLUJO DE SOLICITUD DE CURSOS DE CAPACITACIÓN}

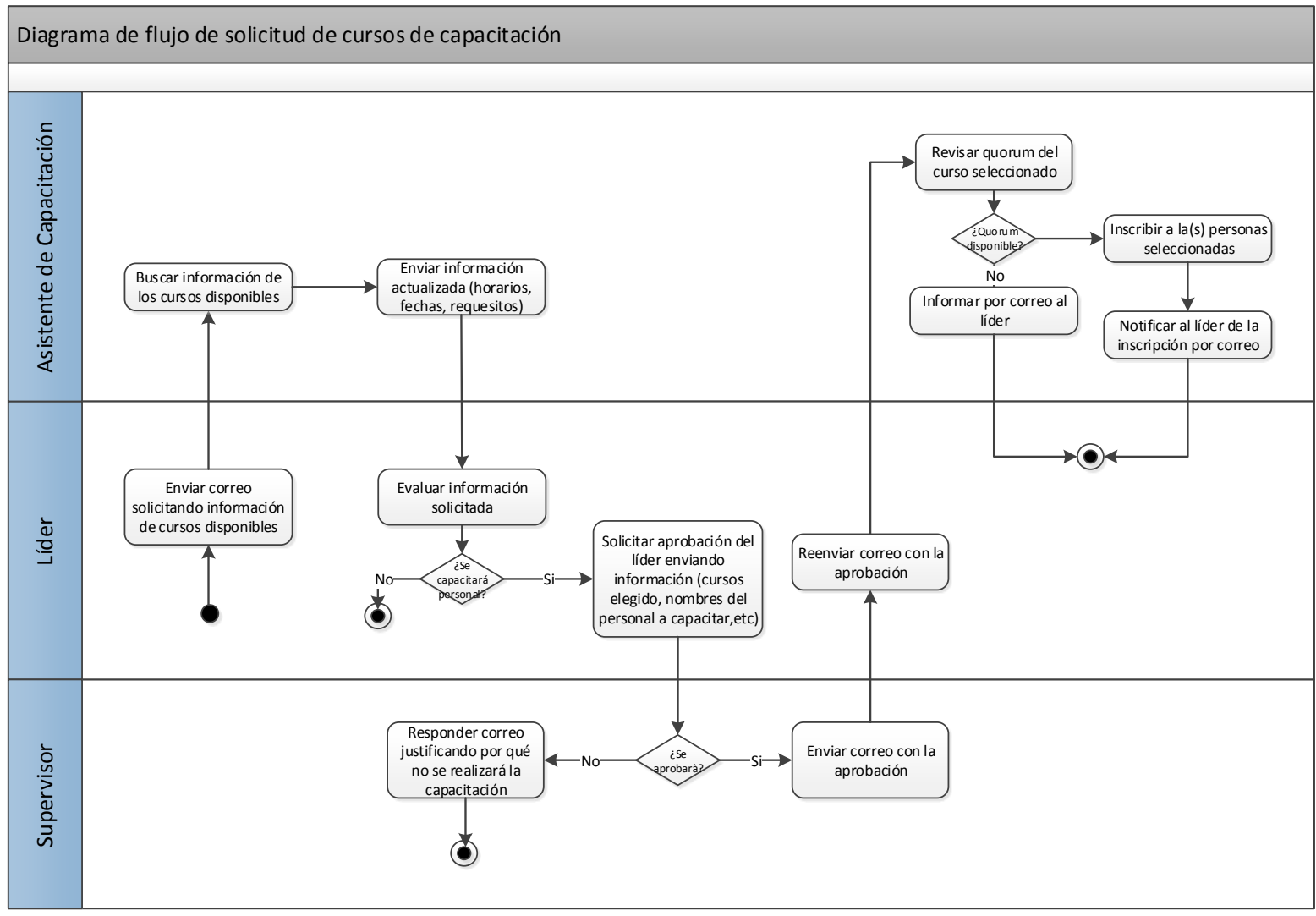




\section{APÉNDICE 4: VISTA DEL WORKFLOW A NIVEL USUARIO}
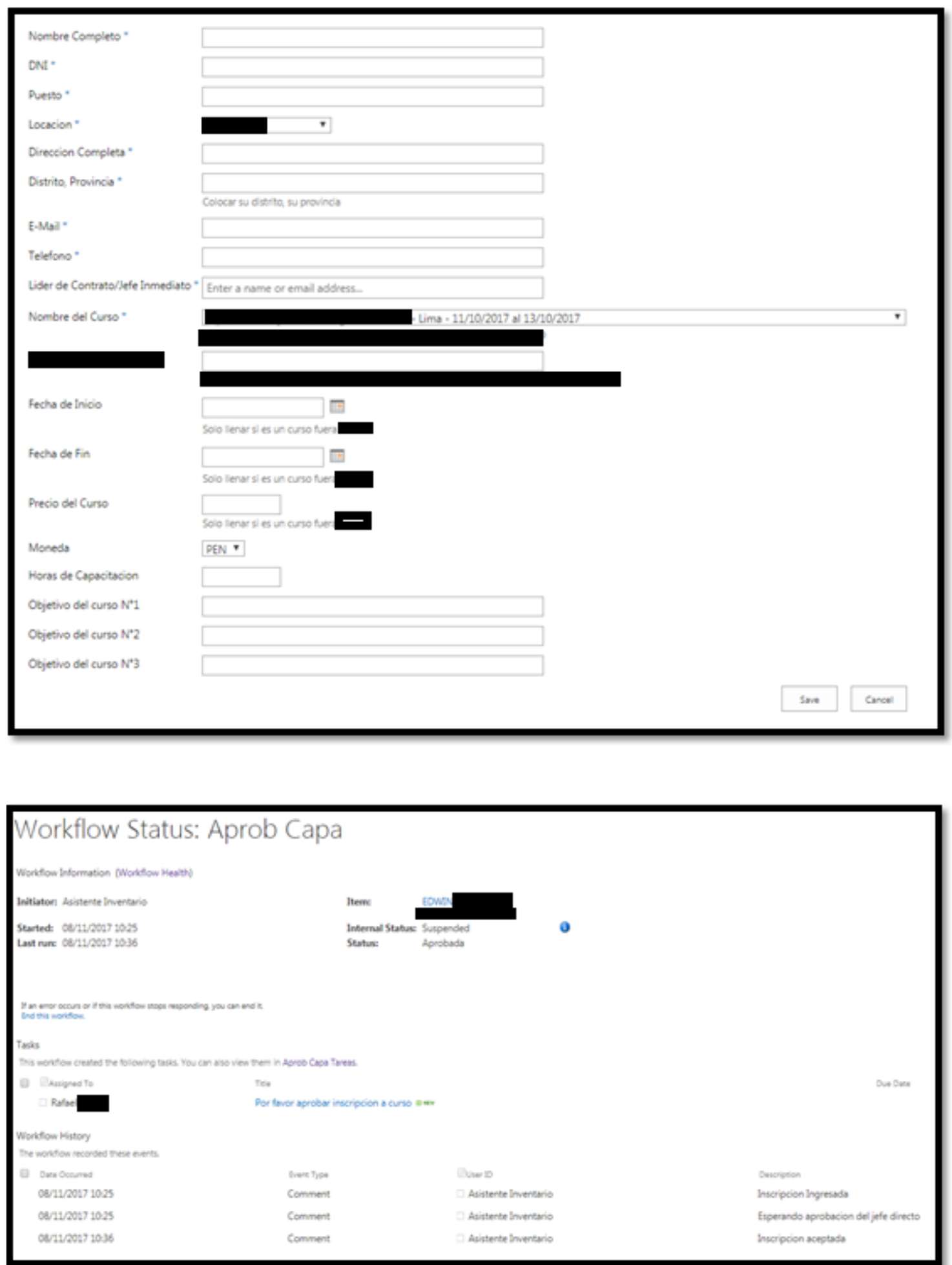


\section{APÉNDICE 5: INDICADORES DE HORAS Y DINERO}

\section{INVERTIDO EN EL 2017}

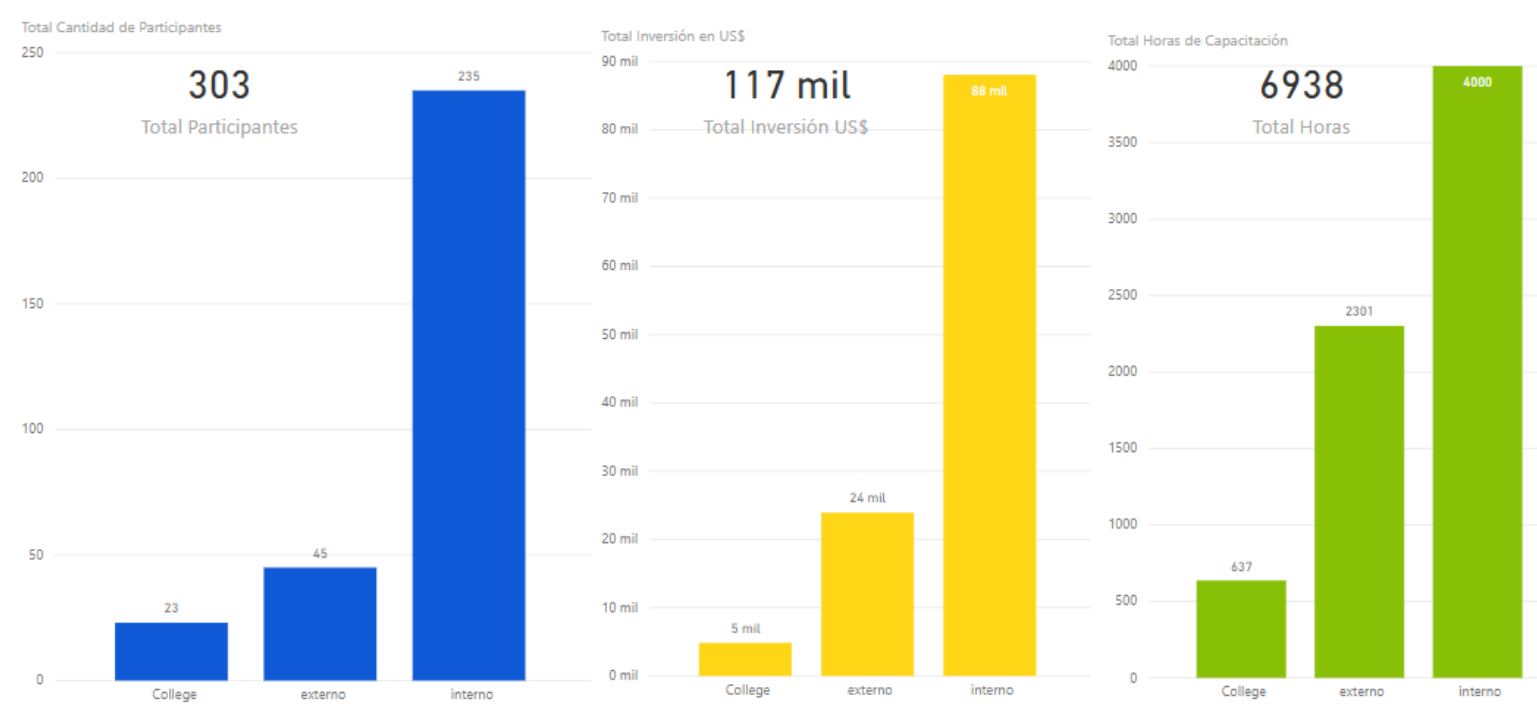

